BULLETIN (New Series) OF THE

AMERICAN MATHEMATICAL SOCIETY

Volume 46, Number 1, January 2009, Pages 99-123

S 0273-0979(08)01233-0

Article electronically published on October 27, 2008

\title{
BIRATIONAL GEOMETRY OLD AND NEW
}

\author{
ANTONELLA GRASSI
}

\begin{abstract}
A classical problem in algebraic geometry is to describe quantities that are invariants under birational equivalence as well as to determine some convenient birational model for each given variety, a minimal model. One such quantity is the ring of objects which transform like a tensor power of a differential of top degree, known as the canonical ring. The histories of the existence of minimal models and the finite generation of the canonical ring are intertwined; minimal models and canonical rings constitute the major building blocks for the birational classification of algebraic varieties. In this paper we will discuss some of the ideas involved, recent advances on the existence of minimal models, some applications, and the (algebraic-geometric proof of the) finite generation of the canonical ring. These results have been long standing conjectures in algebraic geometry.
\end{abstract}

\section{PLAN}

A central problem in many areas of geometry is how to "best" complete an open variety, and also how to understand the relations between the different ways to complete one given variety. In the language of algebraic geometry this becomes constructing a minimal and canonical model and understanding birational equivalence; the construction of the canonical model relies on the finite generation of the canonical ring.

While the existence and meaning of minimal models turns out to be fairly clear in the case of curves, the finite generation is not always obvious (Section 21). In the first part of the last century minimal models were defined and shown to exist for surfaces; a complete classification in birational classes was then later understood (Section 3.1). The question of the finite generation of the canonical ring for surfaces was settled in the early 1960s (Section 3.1.2). The definition of minimal models, which works so well for surfaces, does not make sense in higher dimension: an appropriate generalization was provided in the 1980s, and the "Minimal Model Program" was successfully carried out in dimension 3 (Sections 3.2.1, 3.2.2, 3.3.2).

Since then many partial results have been obtained, and quite a few have turned out to be essential to the proofs of the finite generation of the canonical ring in all dimensions and the existence of minimal models for varieties of general type. It should be said that it is not possible to give here adequate credit to all the people who contributed to the results stated, but there is a brief historical summary at the end of this article. A reference in brackets often merely indicates a paper where a proof could be found (Section 6).

Received by the editors June 8, 2008.

2000 Mathematics Subject Classification. Primary 14E30; Secondary 14J99.

Key words and phrases. Algebraic geometry. 
In $2005 \mathrm{Siu}$ 30] and in 2006 Birkar, Cascini, Hacon, and $\mathrm{M}^{\mathrm{c}}$ Kernan [3] announced the proof of the finite generation of the canonical ring for a smooth variety of general type of any dimension. Birkar, Cascini, Hacon, and $\mathrm{M}^{\mathrm{c}}$ Kernan also deduced that the canonical ring of any smooth variety is finitely generated. We do not discuss here Siu's proof, which is based on analysis. The proof of [3] follows from the authors' results on the existence of minimal models for varieties of general type. Actually, their proof is a clever roundabout induction on the dimension of the variety, which builds on the assumption that finite generation and minimal models exist for varieties of lower dimension. In Section 4 we present an outline of the induction arguments. The proofs of [3] contains quite a few novel ideas, and we try to outline some of them; these include, among others, the Minimal Model Program with scaling.

Section 2 contains examples, facts, and results to introduce algebraic geometry, in particular birational algebraic geometry. Section 3 is an introduction to the Minimal Model Program: we state results, examples, motivations and lingo. Some newer results and applications are in Sections 5.1 and 5.2, which contain applications of the previous results to the classification of algebraic varieties.

In many branches of mathematics classification constitutes an important goal. In high dimensional topology classifications of manifolds of a given homotopy type (such as a sphere or projective space), the classification of three manifolds (such as the classical Poincaré conjecture or the Thurston program), or the classification of finite simple groups are a few well known examples. In algebraic geometry one motivation for the Minimal Model Program is that it provides a powerful tool for the classification of algebraic varieties (or function fields of algebraic varieties); this will be discussed in Section 5.2

In this discussion, we attempt to assume no previous familiarity with algebraic geometry.

\section{ORIGINS OF BIRATIONAL ALGEBRAIC GEOMETRY}

2.1. Some algebraic geometry. The origin of algebraic geometry can perhaps be traced to René Descartes, who noticed that certain geometric objects, namely the graphs of equations, could be studied by combining techniques from algebra and geometry. The fundamental insight is that equations represent relations among different quantities and the representation of these equations in graph form results in either a curve, a surface or an object of higher dimension. When the equations are given in polynomial terms, the corresponding graphs are called algebraic varieties.

The polynomials are taken with complex coefficients, so our varieties will be complex algebraic varieties. In fact the complex numbers are in many ways easier than the real numbers: a complex polynomial of degree $n$ in one variable always has $n$ roots (counted with multiplicity), and differentiable functions have a much tighter structure than infinitely differentiable real functions.

If $f\left(z_{1}, z_{2}\right)$ is a complex polynomial of degree 2 ,

$$
C=\left\{\left(z, 1, z_{2}\right) \in \mathbb{C}^{2} \text { such that } f\left(z_{1}, z_{2}\right)=0\right\}
$$

is an algebraic curve (and a plane conic).

We will consider here projective varieties, that is varieties defined by homogenous polynomials in the projective space $\mathbb{P}^{N}$ : 
Definition 1. $\mathbb{P}^{N}=\left(\mathbb{C}^{N+1} \backslash 0\right) / \mathbb{C}^{*}$, where $\left[a_{0}, \cdots, a_{N}\right]=\left[\lambda a_{0}, \cdots, \lambda a_{N}\right], a_{j} \in \mathbb{C}$, not all zero, and $\lambda \in \mathbb{C}^{*}$; recall that $\mathbb{C}^{*} \stackrel{\text { def }}{=} \mathbb{C} \backslash\{0\}$.

To visualize the complex projective spaces, one can look at the moment map.

Example 2 (The moment map for $\mathbb{P}^{1}$ ). Let $\mu: \mathbb{P}^{1} \rightarrow \mathbb{R}^{2}$ be defined as

$$
\left[z_{0}, z_{1}\right] \rightarrow \frac{1}{\left|z_{0}\right|^{2}+\left|z_{1}\right|^{2}}\left(\left|z_{0}\right|^{2},\left|z_{1}\right|^{2}\right)
$$

The image of $\mu$ is the line segment $I \subset \mathbb{R}^{2}$ (with coordinates $(x, y)$ ) defined by the equation $x+y=1, \quad 0 \leq x \leq 1$. More precisely, $\mu: \mathbb{P}^{1} \rightarrow I$.

It is easy to see that the fibers are circles on the points in the interior of $I$ and a single point over $(0,1)$ and $(1,0) ; \mathbb{P}^{1}$ is then a topological sphere $S^{2}$ :

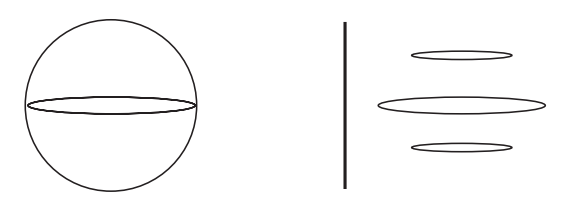

Example 3. $\mathbb{P}^{2} \stackrel{\text { def }}{=}\left(\mathbb{C}^{3} \backslash 0\right) / \mathbb{C}^{*}$. Similarly, let $\mu: \mathbb{P}^{2} \rightarrow \mathbb{R}^{3}$ be defined as

$$
\left[z_{0}, z_{1}, z_{2}\right] \rightarrow \frac{1}{\left|z_{0}\right|^{2}+\left|z_{1}\right|^{2}+\left|z_{2}\right|^{2}}\left(\left|z_{0}\right|^{2},\left|z_{1}\right|^{2},\left|z_{2}\right|^{2}\right)
$$

The image of $\mu$ is a triangle $T \subset \mathbb{R}^{3}$. More precisely, $\mu: \mathbb{P}^{2} \rightarrow T$.

It is easy to see (below) that the fibers are tori on the points in the interior of $T$, circles on interior of the three edges, a single point over the corners:

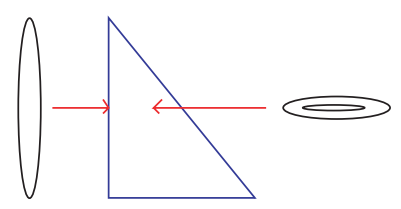

Note that $C \subset \mathbb{P}^{2}$, defined by $\left\{z_{0}=0\right\}$, can be visualized as the $S^{2}$ on the left; $C$ is the locus of coordinates $\left[0, z_{1}, z_{2}\right],\left(z_{1}, z_{2}\right) \in \mathbb{C}^{2}$, which can be identified with the projective line $\mathbb{P}^{1} . C$ is a codimension 1 subvariety, a divisor (see Section 2.1.2. below).

If $X$ is a projective and algebraic variety, then it is also compact (see [31]): the meromorphic functions on $X$ are rational functions and we denote by $\mathbb{C}(X)$ the function field of $X$, the field of all rational functions. The function field of $X$ carries a lot of information about $X$. For example, if a function field of a smooth variety $X$ has a meromorphic function with only a simple pole, then $X$ is necessarily $\mathbb{P}^{1}$. (An algebraic variety is smooth (nonsingular) at a point $P$ if the rank of the Jacobian of the defining polynomials (locally around $P$ ) is maximal. A theorem of Zariski shows that this definition does not depend on the embedding given by the defining polynomials.)

In the Zariski topology the closed sets are the algebraic subvarieties. For example the algebraic variety $Z=\left[0, z_{1}, z_{2}\right] \in \mathbb{P}^{2}$ defined by $z_{0}=0$ is a closed set, its complement $\mathcal{U}=\mathbb{P}^{2} \backslash Z$ is an open set, which can be identified with $\mathbb{C}^{2}$ via the 
map $\left[1, z_{2}, z_{2}\right] \mapsto\left(z_{1}, z_{2}\right) \in \mathbb{C}^{2}$. Note also that $Z$ can be identified with $\mathbb{P}^{1}$. These identifications are examples of isomorphisms:

Definition 4. Let $X$ and $Y \subset \mathbb{P}^{N}$ be projective varieties. $f: X \rightarrow Y$ is a rational map if $f$ can be represented by rational functions $\left(f_{0}, \cdots, f_{N}\right)$. Note that $f$ does not need to be well defined on the whole variety $X$.

Definition 5. $f: X \rightarrow Y$ is a morphism if $f$ is a rational map defined everywhere on $X$.

Note that a rational map $f: X \rightarrow Y$ gives a morphism $f_{\mathcal{U}}: \mathcal{U} \rightarrow Y$ where $\mathcal{U} \subset X$ is a Zariski open set.

Example 6. The map $f: \mathbb{P}^{2} \rightarrow \mathbb{P}^{1}$ defined by $\left[z_{0}, z_{1}, z_{2}\right] \mapsto\left[z_{0}, z_{1}\right]$ is a rational map but not a morphism. In fact, $f([0,0,1])=[0,0]$ is not a point of $\mathbb{P}^{1}$, so $f$ is not defined at $P=[0,0,1]$; on the other hand, $f$ is well defined on the open set $\left\{\mathbb{P}^{2} \backslash P\right\}$ and it is also a morphism.

Definition 7. $f: X \rightarrow Y$ is a birational map if there exist $\mathcal{U} \subset X$ and $\mathcal{V} \subset Y$ Zariski open sets, such that $f_{\mid \mathcal{U}}: \mathcal{U} \rightarrow \mathcal{V}$ is an invertible morphism and the inverse is also a morphism. In this case we write $X \sim Y$, and we say $X$ and $Y$ are birational. $f: X \rightarrow Y$ is a birational morphism if $f$ is as above and $\mathcal{U}=X$. A birational morphism $f$ is called an isomorphism if also $\mathcal{V}=Y$; in this case we write $X \simeq Y$, and we say $X$ and $Y$ are isomorphic.

So $X$ and $Y$ are birational if they have isomorphic open sets.

Definition-Proposition 8 (See for example [12, 31]). The following are equivalent:

(1) $X$ and $Y$ are birational (birationally equivalent);

(2) There exist $\mathcal{U}_{X} \subset X$ and $\mathcal{U}_{Y} \subset Y$, Zariski open sets, such that $\mathcal{U}_{X} \simeq \mathcal{U}_{Y}$;

(3) $X$ and $Y$ have the same function fields.

Example 9. Let $C \subset \mathbb{P}^{2}$ be the cubic curve defined as $z_{3} z_{2}^{2}-z_{1}^{3}=0$; then $f: \mathbb{P}^{1} \rightarrow C$ as $[1, t] \mapsto\left[t^{2}, t^{3}, 1\right]$ is a birational morphism, but it can be shown (see for example, [31]) that it is not an isomorphism.

Example-Definition 10 (A blow up). Let us consider $\mathbb{C}^{2} \times \mathbb{P}^{1}$ with coordinates $\left(\left(x_{0}, x_{1}\right),\left[z_{0}, z_{1}\right]\right)$ and $B=\left\{\left(\left(x_{0}, x_{1}\right),\left[z_{0}, z_{1}\right]\right) \in \mathbb{C}^{2} \times \mathbb{P}^{1}\right.$ such that $\left.x_{j} z_{i}-x_{i} z_{j}=0\right\}$. Then $f: B \rightarrow \mathbb{C}^{2}$ with $\left(\left(x_{0}, x_{1}\right),\left[z_{0}, z_{1}\right]\right) \mapsto\left(x_{0}, x_{1}\right)$ is a birational morphism. Note also that

$$
f_{\left.\right|_{B \backslash f^{-1}(0,0)}}: B \backslash f^{-1}(0,0) \rightarrow \mathbb{C}^{2} \backslash(0,0)
$$

is an isomorphism and that $f^{-1}(0,0) \simeq \mathbb{P}^{1}$.

$E \stackrel{\text { def }}{=} f^{-1}(0,0)$ is called the exceptional divisor of the birational morphism $f$ (see also the introduction to Section 3 ).

The morphism $f: B \rightarrow \mathbb{C}^{2}$ in Example-Definition 10 can be extended to a birational morphism between compact varieties. Let $\widehat{B}$ be the (Zariski) closure of $B$ in $\mathbb{P}^{2} \times \mathbb{P}^{1}$. Then $f$ extends to a morphism $\hat{f}: \widehat{B} \rightarrow \mathbb{P}^{2}$, which restricts us to an isomorphism

$$
\hat{f}_{\left.\right|_{\widehat{B} \backslash \hat{f}^{-1}([0,0,1])}}: \widehat{B} \backslash \hat{f}^{-1}([0,0,1]) \rightarrow \mathbb{P}^{2} \backslash[0,0,1] .
$$

$\widehat{B}$ and $\mathbb{P}^{2}$ are birational. 
Example 11. The blow up of $\mathbb{P}^{2}$ at a point in the previous example can be visualized via the image of the moment map on the right in the picture below. The triangle on the left is the image of the moment map of $\mathbb{P}^{2}$, as in Example 3 , the picture on the right is the image of $\widehat{B}$ under the moment map. The dashed segment on the right is the image, under the moment map, of the exceptional divisor $E$, which we see as a topological sphere $S^{2}$.
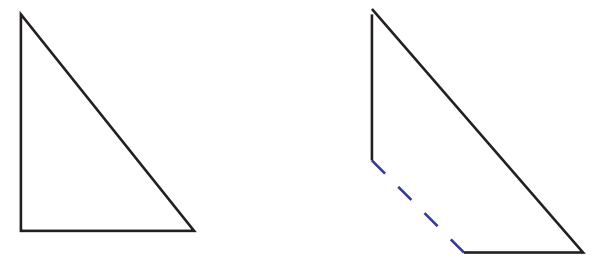

The blow up construction gives two different ways to "complete" the open variety $\mathbb{P}^{2} \backslash[0,0,1]$ to two different compact projective varieties.

Since the above construction is local around $(0,0) \in \mathbb{C}^{2}$, it can be carried through for other surfaces. A similar construction works in all dimensions.

The search for the minimal model can be thought of as the search for the simplest way to birationally complete an open variety, and also to understand the relations between the different minimal models, if more than one model exists. As we will see below and in Section 3, in case of curve and surfaces, it was shown that every birational class has a smooth minimal model 11 It is still not known if any variety has a minimal model; it has been shown that in dimension 3 and higher, the minimal model is not necessarily smooth; the recent paper [3] shows that a minimal model always exists for certain types of varieties (varieties of general type).

A related question is to describe quantities which are invariant under birational morphisms. Before defining some of these invariants (the Kodaira dimension $\kappa(X)$, the plurigenera, the canonical ring), let us see their significance in the case of complex curves.

2.1.1. The case of complex curves: Kodaira dimension and some birational properties. Riemann showed that any compact connected orientable (real) surface can be embedded in a projective space $\mathbb{P}^{N}$, where the image, a complex curve $C$, is defined by a finite set of polynomial equations. The Riemann surfaces are determined up to homeomorphisms by the number $g$ of holes, which is defined to be the genus of the complex curve $C$. It turns out that the genus is a birational invariant.

Theorem 12. $\operatorname{dim}(X)=1$ : If $X$ and $Y$ are smooth and birational, then $X$ and $Y$ are isomorphic.

A couple of remarks are needed here. The theorem does not hold if $X$ and $Y$ are not both smooth, as we saw in Example 9. The theorem does not directly generalize to a higher dimension, as we saw in Example-Definition 10.

The topological Euler characteristic of smooth curves is also a birational invariant; it turns out to be $\chi_{\text {top }}(C)=2-2 g$, which is also the negative of the degree

\footnotetext{
${ }^{1}$ We consider normal varieties; see for example [12]. If $X$ is normal, then the singular locus has at most codimension 2. For example, all normal curves are also smooth, and normal surfaces are singular at most in a set of points.
} 
of the canonical divisor $K_{C}$ (see Example-Definition 14). It also turns out that $2 g-2=\operatorname{deg} K_{C}$ is related to the curvature of the real surface: if the degree is negative, the curvature of the real surface (a sphere) is positive. If the degree is zero, the surface is flat (a torus); if the degree of the canonical divisor is positive, the curvature is negative. Equivalently, the area of a circle of radius $r$ is respectively smaller than, equal to, or bigger than $\pi r^{2}$. The Kodaira dimension is the algebraic analogue of the curvature, and in the above cases we say it is respectively negative, zero, or positive.

2.1.2. Divisors, canonical divisors; canonical rings, finite generation; Kodaira dimension.

Definition 13. An effective Weil divisor $D$ on a variety $X$ is a finite sum $D=$ $\sum_{i} a_{i} D_{i}$ with $a_{i} \in \mathbb{Z}$, where $D_{i}=\left[V_{i}\right]$ are irreducible codimension 1 subvarieties $V_{i}{ }^{2}$ If $a_{i} \geq 0, \forall i, D$ is an effective divisor.

Example-Definition 14. Let $X$ be a complex curve: a divisor $D=\sum_{i} a_{i} D_{i}$ with $a_{i} \in \mathbb{Z}$ is a collection of points. Then $\sum a_{i}$ is the degree of $D$.

Similarly, we can define the degree of a divisor in $\mathbb{P}^{N}$. If a variety $V \subset \mathbb{P}^{N}$ is defined by a polynomial of degree $d, d$ is the degree of the corresponding divisor $[V]$ in $\mathbb{P}^{N}$.

A rational function $f$ on $X$ defines a divisor $(f)$, namely the locus of zeroes and poles counted with multiplicity and sign (the zeroes, positive; the poles, negative); these are the principal divisors. Two divisors are linearly equivalent if their difference is a principal divisor.

Locally on an open set $\mathcal{U}_{j}$, a Weil divisor on a smooth variety is defined by a function $f_{j}$. One could also define a divisor as the collection of these open sets and functions $\left\{\left(\mathcal{U}_{j}, f_{j}\right)\right\}$ with obvious compatibility conditions and equivalence relations. These are called Cartier divisors. One can also view Cartier divisors as invertible sheaves (or line bundles); see for example [12. Multiplication of functions induces tensor powers of the line bundles and sums of divisors. We will use the additive notation: say $m D$ represents the $m$-th tensor power of the line bundle $D$.

When $X$ is not smooth, Weil divisors are not always Cartier divisors, (see Example 58). This is indeed a feature of varieties that are relevant to birational geometry, as we will see in the next sections.

Example 15. If $C \subset \mathbb{P}^{2}$ is a curve, and $H \subset \mathbb{P}^{2}$ a hyperplane, the intersection of $H$ with $C$, denoted as $H \cdot C$, defines a divisor on $C$, which is called very ample.

Before defining a very ample divisor in general we need some more properties. Let $D$ be an effective divisor: $H^{0}(X, D)$ is the set of global sections of $D$, that is the collection of rational functions with at most poles on $D ; H^{0}(X, D)$ is a finite dimensional vector space. Let $\left\{s_{0}, \cdots, s_{N}\right\}$ be a basis of $H^{0}(X, D)$; we can define the rational map

$$
\begin{array}{rr}
\phi_{|D|}: X-\mathbb{P}^{N} \\
p \mapsto & {\left[s_{0}(p), \cdots, s_{N}(p)\right] .}
\end{array}
$$

This map depends only on the linear equivalence class of the divisor $D$.

\footnotetext{
${ }^{2} V_{i}$ is irreducible if it is not reducible. A variety $A$ is reducible if there exist varieties $B$ and $C$ such that $B \cup C=A$ with $B \neq A$ and $C \neq A$.
} 
Definition 16. Let $X$ be an algebraic variety and $D$ a divisor on $X . D$ is very ample if $\phi_{|D|}: X \hookrightarrow \mathbb{P}^{N}$ is an embedding 3 In particular $D$ is the restriction of a hyperplane $H \subset \mathbb{P}^{N}$ to $X$; we write $D \stackrel{\text { def }}{=} \phi^{*}(H)$.

It is a crucial problem in this setting (see Section 3.2) to identify a priori which divisors (or their multiples) are very ample and which divisors (or their multiples) determine morphisms, not necessarily embeddings.

Definition 17. Let $X$ be an algebraic variety and $D$ a divisor on $X ; D$ is base point free if there exists a morphism $\phi_{|D|}: X \rightarrow \mathbb{P}^{N}$.

Definition 18. Let $X$ be an algebraic variety and $D$ a divisor on $X ; D$ is semiample if there exists some $m>0$ such that $m D$ is base point free.

A canonical form on $\mathbb{C}^{n}$, with coordinates $\left(z_{1}, \cdots, z_{n}\right)$ is a top differential form, for example $d z_{1} \wedge \cdots \wedge d z_{n}$. On a general smooth variety $X$, the canonical form is a collection of volume forms on open sets with obvious compatibility conditions. The canonical divisor is the divisor naturally associated to it and denoted by $K_{X}$; the canonical divisor is defined up to linear equivalence.

In some cases the canonical divisor can be defined even when a variety $X$ is singular, as in the surface below.

Example 19 (Kummer surface). Let $X=(E \times E) /\langle\imath, \mathrm{id}\rangle$, where $E$ is the torus in Example 28 and $\imath:\left(z_{1}, z_{2}\right) \mapsto\left(-z_{1},-z_{2}\right)$ with $\left(z_{1}, z_{2}\right) \in \mathbb{C}^{2} . X$ has 16 singular points, and the canonical differential on $E \times E$ is preserved by the group action and induces a canonical divisor on $X$. The canonical divisor on $X$ is still trivial: $H^{0}\left(X, K_{X}\right) \simeq \mathbb{C}$.

It is of particular interest, in the context of birational geometry, to understand under which conditions on a variety $X$ the canonical divisor $K_{X}$ is semiample, as we will see below in Example-Theorem 29, Theorem 37, and Section 5.2. Also of importance is the space of sections $H^{0}\left(m K_{X}\right)$.

Definition 20. $\operatorname{dim} H^{0}\left(X, m K_{X}\right), m \geq 0$, is called the m-plurigenus of $X$.

The following proposition holds:

Proposition 21. The plurigenera $\operatorname{dim} H^{0}\left(X, m K_{X}\right)$ are birational invariants.

The algebraic definition of the genus of a smooth curve $X$ is $g(X)=$ $\operatorname{dim} H^{0}\left(X, K_{X}\right)$, which coincides with the topological definition of the genus, as we will see in Examples 27 and 28 and Example-Theorem 29 below.

Definition 22 (Mumford, [27]). $R\left(X, K_{X}\right)=\bigoplus_{m \geq 0} H^{0}\left(m K_{X}\right)$ is the canonical ring.

Proposition 21 implies that the canonical ring is a birational invariant. We assume $X$ to be connected, hence $H^{0}\left(X, 0 \cdot K_{X}\right)=\mathbb{C}$.

Definition 23. The Kodaira dimension $\kappa(X)$ of a variety $X$ is defined as follows: $\kappa(X)<0$ if $H^{0}\left(X, m K_{X}\right)=0, \forall m>0$; $\kappa(X)=k$, if $\operatorname{dim} H^{0}\left(X, m K_{X}\right) \sim m^{k}$ as $m \rightarrow \infty$.

\footnotetext{
${ }^{3}$ That is, $\phi(X)$ is a closed subvariety of $\mathbb{P}^{N}$ isomorphic (via $\phi$ ) to $X$.
} 
Note that $\kappa(X)<0$ is also denoted by $\kappa(X)=-1$ or $\kappa(X)=-\infty$. Obviously, $\kappa(X) \leq \operatorname{dim}(X)$; it turns out that

$$
\kappa(X)=\text { transc. } \operatorname{deg} R\left(X, K_{X}\right)-1 .
$$

Definition 24. $X$ is of general type if $\kappa(X)=\operatorname{dim}(X)$. If $X$ is a variety of general type, $K_{X}$ is big.

Similarly, one defines $\kappa(X, D)$, the Kodaira dimension of a divisor $D$. If $\kappa(X, D)=$ $\operatorname{dim}(X), D$ is called big and $D$ is of general type.

There are many varieties of general type. For example the curves of general types are the curves with genus $g \geq 2$; see the examples below. Any hypersurface defined by polynomials of degree greater than $d+1$ in $\mathbb{P}^{d}$ is also of general type. This can be seen from the adjunction formula:

Proposition 25. If $V \subset X$ is a smooth hypersurface in a smooth variety $X$, then

$$
K_{V}=\left(K_{X}+V\right)_{\mid V} .
$$

We now see some examples of the results discussed above in the case of curves:

Remark 26. If $X$ is a smooth curve

$$
K_{X}=\Omega_{X}^{1}=T_{X}^{*} \text { and } \chi_{\text {top }}(X)=2-2 g=-\operatorname{deg}\left(K_{X}\right),
$$

where $T_{X}, T_{X}^{*}$ denote the tangent and cotangent bundle, respectively. Note also that $c_{1}(X)=-K_{X}$. Then $K_{X}$ is noneffective (we write $K_{X}<0$ ) if and only if $g=0, K_{X}$ is trivial (we write $K_{X} \simeq 0$ ) if and only if $g=1$, and $K_{X}$ is effective (we write $K_{X}>0$ ) if and only if $g \geq 2$.

Example 27 (Genus $g=0$ ). If $X=\mathbb{P}^{1}$, on the open set $\mathcal{U}_{1} \simeq\{[s, 1], s \in \mathbb{C}\}$ the local volume form is $d s$, if $\mathcal{U}_{2} \simeq\{[1, t]\}$, on $\mathcal{U}_{1} \cap \mathcal{U}_{2} s=1 / t$ and the local volume form is $d s=d(1 / t)=-1 / t^{2} d t$. The canonical divisor (Weil) corresponds to a pole with multiplicity 2 ; it is not an effective divisor, but its inverse is. In this case, $H^{0}\left(\mathbb{P}^{1}, K_{X}\right)=0, g(X)=0, \kappa(X)<0$, and the canonical ring is trivial.

Example 28 (Genus $g=1$ ). The torus $E=\mathbb{C} / \mathbb{Z} \oplus \imath \mathbb{Z}$ can be given the structure of an algebraic curve $X$, which can be identified with a smooth cubic in $\mathbb{P}^{2}$. It is easy to see that the volume form on $\mathbb{C}, d z_{1}$ induces a global volume form $X$. The divisor associated to it has neither poles nor zeroes, it is the trivial divisor. Here $H^{0}\left(E, K_{X}\right)=\mathbb{C}, g(X)=1, \kappa(X)=0$, and the canonical ring is $R\left(X, K_{X}\right)=$ $\bigoplus_{m} \mathbb{C}$.

Example-Theorem 29 (The tricanonical embedding, [Riemann]). (See for example [12] or [31.) Riemann showed that if $C$ is a curve of genus $g \geq 2$, then $3 K_{C}$ is very ample, that is there is an embedding $\phi_{\left|3 K_{C}\right|}: C \hookrightarrow \mathbb{P}^{N}$ with the following property. If $H \subset \mathbb{P}^{N}$ is a hyperplane, then $H \cdot C$ is $3 K_{C}$. Then the canonical ring $R\left(C, K_{C}\right)$ can be reconstructed 4 from the coordinate ring of $\mathbb{P}^{N}$. In particular, $R$ is finitely generated (see below). Note that the pluricanonical embedding determined by $3 K_{C}$ gives us an explicit description of $C$ as an algebraic subvariety in a projective space; in fact, $C \simeq \operatorname{Proj} R\left(C, K_{C}\right)$ (see Remark 30). Note also that $X$ is of general type.

\footnotetext{
${ }^{4}$ In fact Serre's vanishing theorem for the first cohomology of the ideal sheaf of $C$ in $\mathbb{P}^{N}$ (twisted by $m$ ) shows that there is a surjection $H^{0}\left(\mathbb{P}^{N}, m H\right) \rightarrow H^{0}\left(C, 3 m K_{C}\right)$.
} 
Remark 30. We refer to 12 for a formal definition of $\operatorname{Proj}(R)$, for $R$ a finitely generated ring. If $R$ is a finitely generated ring over $\mathbb{C}$, the relations among the generators in $\mathbb{C}\left[x_{0}, \cdots, x_{N}\right]$ (or better the ideal generated by the relations) define an algebraic variety $X \subset \mathbb{P}^{N}$. Then $X \stackrel{\text { def }}{=} \operatorname{Proj}(R) ; R$ is the homogeneous coordinate ring of $X$ (see also [12]).

A nice discussion of Proj in the context of birational geometry can be found in Section 6 of [19]. Examples 27 and 28, and Example-Theorem 29] show the following theorem.

Theorem 31. The canonical ring $R$ of a smooth algebraic curve $X$ is always finitely generated. In addition, if $X$ is of general type, then $X=\operatorname{Proj}(R) . X=\operatorname{Proj}(R)$ is called the canonical model.

In the case of surfaces, it is much harder to show that the canonical ring is finitely generated; this will be discussed in the next section.

\section{Minimal models? Canonical models? An introduction}

We have seen that a birational morphism between algebraic varieties $X$ and $Y$ is a map that is an isomorphism between some Zariski open sets. Zariski open sets are dense in algebraic varieties, and the existence of a birational morphism between $X$ and $Y$ implies strong relations between them. In the case of curves there is a unique smooth projective curve in each birational equivalence class and the canonical ring $R$ is finitely generated. In addition if a curve is of general type, $R$ gives an explicit embedding of the curve, the canonical model.

It is natural to ask if similar statements also hold for surfaces, threefolds, and higher dimensional varieties. We have seen in Example-Definition 10 that $\mathbb{P}^{2}$ and $\widehat{B}$ are smooth and birational surfaces, and that one can construct infinitely many smooth projective surfaces in each birational equivalence class. So it is natural to ask if, in each birational class, we can identify a simpler model(s), a minimal model, and how we can produce it. It is also natural to ask if the canonical ring is finitely generated and if it gives an embedding for varieties of general type.

Zariski [36] first studied the ring $R=\bigoplus_{m} H^{0}(m D)$, for a divisor $D$ on a surface $X$, he also showed that $R$ is not always finitely generated, for some particular divisors $D$. Much later, Wilson [35] gave examples of varieties where the canonical ring is not finitely generated: in these examples the varieties are not algebraic or they have worse singularities than the one considered here.

We will see that in the case of surfaces the canonical ring $R$ and its finite generation can be instrumental to the understanding of a given variety (see Remark 40). The proof of the finite generation of the canonical ring for surfaces was one of the motivations for the birational classification of surfaces, which was then completed on the basis of the existence of minimal models. Also, the proof of finite generation relies on the existence of minimal models.

In higher dimension the canonical model $\operatorname{Proj}(R)$ turns out to be a crucial ingredient to the understanding of birational geometry of varieties, and the existence of minimal models and canonical models becomes interdependent and intertwined. In particular, in the arguments of [3] and [11, the existence of canonical and minimal models depend on each other in the ascending induction arguments.

In the first part of the 20th century Castelnuovo, Enriques, and Severi gave an algorithm to construct a minimal model of a given smooth surface; the definition 
and the algorithm are specific to dimension 2. By the middle 1980s a proposal for a Minimal Model Program in higher dimension had been formed and also carried out in many aspects, by the work of Clemens, Kawamata, Kollár, Miyaoka, Mori, Reid, Shokurov, Viehweg and many others (see [19] and 23]). The Minimal Model Program was successfully completed by Mori [26] for $\operatorname{dim} X=3$ by the end of the 1980s. It was also understood how different minimal models of threefolds are related (see again [19] and [23).

3.1. The case of surfaces. The results stated in this section are by now called classical; we discuss them in detail because they illustrate some of the basic ideas which recur in higher dimension, without many of the complications. On the other hand the presentation we give here is not the classical one developed by the Italian school, which is in fact specific to dimension 2 only.

3.1.1. Minimal models, following the Italian school. Proofs for the results stated in this section can be found in many books; here we mostly follow [1] and 23.

In Example-Definition 10 we noted that any smooth point on a surface can be blown up to obtain another smooth, birationally equivalent surface. The Italian school came up with the following definition and proved the theorems below:

Definition 32 (Classical). A smooth surface $\bar{S}$ is minimal if any birational morphism $\psi: S \rightarrow T$ to another smooth surface $T$ is an isomorphism.

Theorem 33. Any smooth surface $S$ has a minimal model.

Proof. If any birational morphism $S \rightarrow T$, with $T$ a smooth surface, is an isomorphism, then $S$ is minimal and we are done. If $S$ is not minimal, we can find a sequence of birationally equivalent smooth surfaces $\left\{S_{j}\right\}, j=1, \cdots, \ell$, with $S_{\ell}$ minimal. In fact, if $S$ is not minimal by definition there exists a smooth surface $S_{1}$ and a birational morphism $\varphi_{1}: S \rightarrow S_{1}$ which is not an isomorphism; it is not hard to see that $b_{2}\left(S_{1}\right)<b_{2}(S)$, where $b_{2}$ is the second Betti number (see, for example, [1]). If $S_{1}$ is minimal, we are done; otherwise, we find another smooth surface $S_{2}$ birationally equivalent to $S$ and $b_{2}\left(S_{2}\right)<b_{2}(S)$. Thus we get a collection of morphisms $\varphi_{j}: S_{j-1} \rightarrow S_{j}$ and smooth surfaces $S_{j}$ that are birationally equivalent to $S$. The process must stop after a $\ell$ number of steps, since $b_{2}\left(S_{j}\right)<b_{2}\left(S_{j+1}\right)$, $\forall j$.

Furthermore the following holds:

Theorem 34. Let $S$ be a smooth surface:

a: If $\kappa(S) \geq 0$, then the minimal model of $S$ is unique.

b: If $\kappa(S)<0$, the minimal model is not unique. In this case $S$ is birationally equivalent to $\mathbb{P}^{1} \times C$, for some smooth curve $C$, and we say that $S$ is birationally ruled.

It is not hard to see, for example, that $S=\mathbb{P}^{1} \times \mathbb{P}^{1}$ and $T=\mathbb{P}^{2}$ are both minimal surfaces, according to Definition 32, with $\kappa(S)=\kappa(T)<0 . S$ and $T$ are also birational, as the blow up of $S$ at one point is isomorphic to the blow up of $T$ at two different points.

Definition 32 and Theorem 33 do not provide an explicit algorithm for constructing a minimal model for a given smooth surface; the basic tool for such an algorithm is given by Castelnuovo's contraction criterion, which is the building block of the 
classical birational theory of surfaces. Castelnuovo's theorem provides a necessary and sufficient condition for a smooth curve $E$ on a smooth surface $S_{1}$ to be the exceptional divisor of a blow up $\varphi: S_{1} \rightarrow S_{2}$ :

Theorem 35 (Castelnuovo). Let $S_{1}$ be a smooth surface and $E$ a smooth rational curve on $S_{1}$ with $E^{2}=-1$. Then there exists a birational morphism $\varphi: S_{1} \rightarrow S_{2}$, where $S_{2}$ is a smooth surface; $\varphi$ is the blow up of $S_{2}$ with exceptional divisor $E$ (as in Example-Definition (10).

The statement of this theorem is specific to surfaces: the exceptional divisors are curves only in the case of surfaces and the "negative self-intersection" makes sense only in dimension 2. In Section 3.2.1 we revisit these theorems (and definition), in light of the Minimal Model Algorithm, which makes sense in all dimensions.

3.1.2. Canonical models, finite generation of the canonical ring. The CastelnuovoEnriques-Kodaira classification then provides a finer structure theory for the surfaces that are not birationally ruled, which we discuss briefly at the end of this section. The classification is used in the proof of the theorem below, following [1]:

Theorem 36 (Mumford, 1962). The canonical ring of an algebraic surface $Z$ is finitely generated.

Proof (Sketch). Let $S$ be a minimal model of $Z$ (see Section 3.1.1). If $\kappa(S)<0$, then the canonical ring of $S$ is finitely generated, as in the case of $\mathbb{P}^{1}$ (Example 27). Recall that $\kappa(S) \leq \operatorname{dim}(S)=2$. If $\kappa(S)=0$, then the birational classification implies that $n K_{S} \simeq \mathcal{O}_{\mathcal{S}}$ for some $\mathrm{n}$, so the canonical ring is again finitely generated, as in the case of elliptic curves (Example 28). If $\kappa(S)=1,2$ then it can be shown that $K_{S}$ is semiample (see Definition [18), and we can deduce that the canonical ring is finitely generated, as in the case of curves of general type (Example-Theorem (29).

The proof sketched above is not Mumford's original proof, as it uses the classification of surfaces; the completion of this classification was in fact spurred by Mumford's theorem.

Following Mumford, Bombieri showed:

Theorem 37 (2]). Let $S$ be a minimal surface of general type. Then $m K_{S}$ is base point free (see Definition 17) for $m \geq 4$, and if $m \geq 5$, the morphism is birational.

Remark 38. Let $\phi_{\left|m K_{S}\right|}: S \rightarrow W_{m}$ be the morphism determined by $m K_{S}, m \geq 5$ as above. It follows that the $W_{m} \simeq \operatorname{Proj}\left(R\left(S, K_{S}\right)\right)$ for all the relevant $m ; X^{\text {can }} \stackrel{\text { def }}{=} W_{m}$ is called the canonical model of $S$. The canonical model is unique.

Remark 39. If $\phi_{\left|m K_{S}\right|}: S \rightarrow X^{\text {can }}$, as in Remark 38, is not an isomorphism, then $S$ can be seen as a minimal resolution of the canonical model $X^{\text {can }}$. The singularities on $X^{\text {can }}$ are called canonical (see Theorem-Definition 61).

The two dimensional canonical singularities are also known as rational double points, and they are locally of the form $\mathbb{C}^{2} / \Gamma$, where $\Gamma \subset \mathrm{SL}(2, \mathbb{C})$ is a finite subgroup. The singularities in Example 19 are canonical.

Remark 40. The morphisms determined by the canonical divisor $K_{S}$ (or its multiples $m K_{S}$ ) on surfaces of general type were studied by Enriques [7, as a crucial tool for understanding these surfaces; see a nice exposition in [4]. 
Another strategy to construct the minimal model for a surface $S$ of $\kappa(S)=2$ could be to consider the canonical model $X^{\text {can }}$ and its minimal smooth resolution (which is knows to exist).

Part $\mathbf{b}$ in Theorem 34, together with Theorem 41 below provides a "structure" theorem for surfaces of negative Kodaira dimension and $\kappa(S)=1$ :

Theorem 41. Let $S$ be a minimal surface with $\kappa(S)=1$. The canonical divisor $K_{S}$ is semiample and, for some $m \gg 0$ there is a morphism $\phi_{\left|m K_{S}\right|}: S \rightarrow B$, where $B=\operatorname{Proj}\left(R\left(S, K_{S}\right)\right.$ ). The general fiber is a curve $C$ with $\kappa(C)=0$ (an elliptic curve).

The fibration in the above theorem is called Iitaka's fibration; see Theorem 90

3.2. Towards higher dimensions. The Castelnuovo-Enriques classification was completed by the middle of the 19th century. For a long time the proof of the finite generation of the canonical ring and a birational classification in higher dimension seemed out of reach. One of the major obstacles was the lack of a "good" analogue of the notion of minimal model; in fact the classical Definition 32 does not make sense in dimension 3 (see Example 86).

3.2.1. Minimal models for surfaces, towards higher dimension. The starting point is the following observation:

Remark 42. Castelnuovo's Theorem 35] says that a birational morphism of smooth surfaces $f: S \rightarrow \bar{S}$ which is not an isomorphism contracts (to points) rational curves $E$ on $S$ such that $K_{S} \cdot E<0$. This follows from the adjunction formula on surfaces, see Proposition 25.

Definition 43. A divisor $D$ on a variety $S$ is nef if $D \cdot C \geq 0$, for all the effective curves $C$ in $S$.

The following holds:

Theorem 44. If $S$ is not birationally ruled and $K_{S}$ is not nef, then there exists a rational exceptional curve as in Castelnuovo's criterion.

We will not discuss here the proof of these results, which is related to both the existence of rational curves when $K_{S}$ is not nef and the "bend and break" arguments (see [19, Chapter 1.1).

Definition 45 (Revised). A smooth surface $S$ is minimal if $K_{S}$ is nef.

This definition makes sense in all dimensions. So the key idea of Mori's algorithm in all dimensions is to contract all curves $C$ such that $K_{X} \cdot C<0$. We will see that contracting such curves will also provide a structure theorem for birationally ruled varieties (the variety of negative Kodaira dimension), as in the following cases:

Example 46 (Trivial, but useful remark).

0: If $S=\mathbb{P}^{2}$, then there exists a trivial morphism $\pi: \mathbb{P}^{2} \rightarrow\{p t$.$\} (the fiber of$ $\pi$ is the whole $\mathbb{P}^{2}$ ).

1: If $S=\mathbb{P}^{1} \times \mathbb{P}^{1}$, then there exists a fibration $\pi: S \rightarrow \mathbb{P}^{1}$ (the projection on one factor), with fibers $\mathbb{P}^{1}$ (the other factor).

We still need an analogue of Castelnuovo's Theorem 35 that works in all dimensions. We will need the definitions of the following section. 
3.2.2. The Mori cone; ample, nef, pseudo-effective cones and big divisors. More details on the definitions and results stated here can be found, for example, in [19], [15], 23].

A 1-cycle is a linear combination of subvarieties of dimension 1 in a variety $X$ :

Definition 47. Let $X$ be a smooth variety. Let $N E(X)$ be the cone generated by classes of effective 1-cycles, with real coefficients, and let $\overline{N E(X)}$ denote its closure. $\overline{N E(X)}$ is the Mori cone of $X$.

We consider the Mori cone modulo numerical equivalence (which means from the point of view of intersection with divisors, they behave the same).

Definition 48. A divisor $D$ is called ample if $m D$ is very ample (see Definition 16), for some $m \in \mathbf{N}$.

Theorem 49 (Kleiman's criterion). $D$ is ample if and only if $D \cdot C>0$, for every nonzero curve $C \in \overline{N E(X)}$.

We consider the cone of ample divisors and the cone of nef divisors, modulo numerical equivalence (which means from the point of view of intersection with curves, they behave the same).

Remark 50. The nef cone and the Mori cone are then dual cones with respect to the intersection pairing. The closure of the ample cone is the nef cone.

It is also useful to consider the cone of effective divisors, with real coefficients, modulo numerical equivalence. Its closure is called the pseudo-effective cone; a divisor $D$ in the closure is called pseudo-effective. These divisors appear in more general versions of the statements of 3 than we discuss in Section 4 , with the exception of Theorem 89] The following result is also used.

Proposition 51. A big divisor can be written as the sum of an ample divisor and an effective divisor.

Note that the following four inclusions of the cones hold:

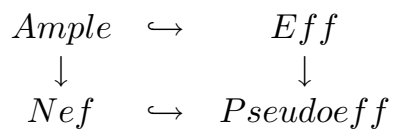

Definition 52. A ray $R$ in $\overline{N E(X)}$ is defined as $R=\mathbb{R}^{+}[Z]$, for some $Z \in \overline{N E(X)}$. The ray $R$ is extremal if $z_{1}+z_{2} \in R \Rightarrow z_{1}, z_{2} \in R$ (in the sense of convex geometry), and $K_{X} \cdot R<0$.

Example 53 (See, for example, 19]). Let $S$ be a smooth surface and $E$ a rational curve with $E^{2}=-1$. Then $E$ generates an extremal ray.

Example 54 (See, for example, [19] and Example 46). Let $S=\mathbb{P}^{2}$. Then the class of a line $L$ is an extremal ray.

Example 55 (See, for example, [19] and Example 46). Let $S=\mathbb{P}^{1} \times \mathbb{P}^{1}$. Then the class $F$ of a factor $\mathbb{P}^{1}$ is an extremal ray.

Definition 56. Let $R$ be an extremal ray. A Cartier divisor $D$ is the supporting divisor of $R$ if and only if $D \cdot Z \geq 0$, for all $Z \in \overline{N E(X)}$ with equality if and only if $Z \in R$. 
Geometrically, the hyperplane defined by the supporting divisor $D$ intersects the cone $\overline{N E(S)}$ only on the ray $R$. This is a necessary condition for a morphism $\phi_{|m D|}$ (if it exists) to contract exactly the curves in the ray $R$. Castenuovo's contraction theorem shows that there exists a semiample divisor $D$ (that is $m D$ is base point free for sufficiently large integers $m$ ), such that $\phi_{|m D|}: X \rightarrow X_{1}$ contracts exactly the extremal ray generated by the rational exceptional curve $E$ with $E^{2}=-1$. The morphisms in Example 46 contract exactly the extremal rays $L$ and $F$.

To generalize Castelnuovo's criterion, we need to find a supporting divisor $D$ for an extremal ray $R$ such that $D$ is semiample. Note that this is what happens also for the morphisms in Example 46 .

We will need some definitions and properties.

3.3. Higher dimensions: Minimal modes, finite generation, canonical models. It is in higher dimension that minimal models, canonical models, and finite generation get intertwined.

In dimension 3 or higher one needs to allow certain mild singularities:

3.3.1. Singularities. In fact Mori [25] showed that if $\operatorname{dim}(X) \geq 3$, contracting certain curves $C$ on extremal rays on smooth varieties produces singularities on the image variety. The nice thing is that the program also works with these singularities, and these are called $(\mathbb{Q}$-factorial-)terminal singularities.

Definition 57. A Weil divisor $D$ on a normal variety is called $\mathbb{Q}$-Cartier (resp. $\mathbb{R}$ Cartier) if $m D$ is Cartier for some $m>0, m \in \mathbb{Q}$ (resp. $m \in \mathbb{R}) . X$ is $\mathbb{Q}$-factorial if every Weil divisor is $\mathbb{Q}$-Cartier. Let $D$ and $E$ be $\mathbb{Q}$ - (resp. $\mathbb{R}$-) Cartier divisors. $D$ and $E$ are equivalent if there exists a $m$ such that $m D$ and $m E$ are equivalent divisors. If $C$ is a curve, we also define

$$
D \cdot C \stackrel{\text { def }}{=} \frac{1}{m}(m D \cdot C) .
$$

Note that the definitions in Section 3.2.2 can be applied also to $\mathbb{Q}$-divisors, and $\mathbb{R}$-divisors. In fact in the proofs in 3 the use of $\mathbb{R}$-divisors is essential.

Example 58. Let $X=\mathbb{C}^{2} / \tau$, where $\tau=\left\langle e^{\pi i / 3}, e^{2 \pi i / 3}, 1\right\rangle$ and $\tau:\left(z_{1}, z_{2}\right) \mapsto$ $\left(e^{\pi i / 3} z_{1}, e^{\pi i / 3} z_{2}\right) . X$ is singular at $(0,0)$, but the canonical differential on $\mathbb{C}^{2}$ is not preserved by the group action. However, the third tensor power of the canonical differential is, and it induces a Cartier divisor $3 K_{X}$ on $X . K_{X}$ is then a $\mathbb{Q}$-Cartier divisor.

Theorem-Definition 59. $X$ has at most terminal singularities if $K_{X}$ is $\mathbb{Q}$-Cartier and for every (equivalently, for any) resolution $h: \widetilde{X} \rightarrow X$, we have $m K_{\widetilde{X}}=$ $h^{*}\left(m K_{X}\right)+F$, for some effective divisor $F$ supported on the exceptional locus and every irreducible component of the exceptional locus appears in $F$ with strictly positive multiplicity.

It is not hard to see that a surface $S$ has at most terminal singularities if it is smooth; the singularities of the surface canonical models (see Remark 39) satisfy this similar property:

Proposition 60. Let $X^{\text {can }}$ be the canonical model of a surface of general type and $\phi: S \rightarrow X^{\text {can }}$ the minimal resolution as in Theorem 37. Then $K_{S}=\phi^{*}\left(K_{X^{\text {can }}}\right)$.

The definitions can be combined as follows. 
Theorem-Definition 61. $X$ has at most canonical singularities if $K_{X}$ is $\mathbb{Q}$ Cartier, and for every (equivalently, for any) resolution $h: \widetilde{X} \rightarrow X$, we have $m K_{\widetilde{X}}=h^{*}\left(m K_{X}\right)+F$, for some effective divisor $F$ supported on the exceptional locus. If every irreducible component of the exceptional locus appears in $F$ with strictly positive multiplicity, then $X$ is said to have at most terminal singularities.

It can be shown that canonical and terminal singularities do not affect the plurigenera, hence the canonical ring. This property was in fact a motivation for considering this class of singularities (see [28, [29]).

Now we can state the higher dimensional definition of a minimal (and canonical) model

Definition 62. $X$ is a canonical (resp. minimal) model if $X$ has at worst canonical (resp. terminal ) singularities and $K_{X}$ is an ample (resp. nef) $\mathbb{Q}$ Cartier divisor.

and the generalization of Castelnuovo's criterion:

Theorem 63 (The contraction theorem). Let $X$ be a projective variety with at worst canonical singularities. Then every extremal ray $R$ has a supporting semiample divisor.

Key ingredients in the proof of the above theorem are the Vanishing Theorems, the Nonvanishing Theorem, the Cone Theorem, the Rationality Theorem, and the Basepoint Free Theorem (see, for example, [19] or [23]).

Theorem-Definition 64 (Kawamata-Shokurov). If $\bar{X}$ is a minimal model of a projective variety $X$ and $K_{X}$ is also big, then $K_{\bar{X}}$ is semiample, and the canonical ring is finitely generated. Then $X^{\mathrm{can}}=\operatorname{Proj}\left(R\left(X, K_{X}\right)\right)$ is called the canonical model of $X$, and the singularities are canonical.

Recall that the canonical model is unique. Now we can see the Minimal Model Algorithm at work:

Theorem $65([26])$. Let $X$ be a projective variety with only $\mathbb{Q}$-factorial terminal singularities. If $X$ is not a minimal model, then there exists a surjective morphism $f: X \rightarrow Y$ to a normal projective variety $Y$ with connected fibers, and one of the following holds:

(1) $\operatorname{dim}(X)>\operatorname{dim}(Y)$ (Mori fiber space).

(2) $f$ is birational and contracts a divisor (divisorial contraction).

(3) $f$ is birational and contracts no divisor (small contraction).

Furthermore:

In Case $(1) \kappa(X)<0$.

In Case (2) $Y$ has at most terminal singularities.

In Case (3) $Y$ is not $\mathbb{Q}$-factorial.

It is useful to see the algorithm at work in the case of surfaces; we will not discuss this here (see for example 23]). Case (2) is Castelnuovo's Theorem $35 \mathbb{P}^{2}$ and $\mathbb{P}^{1} \times \mathbb{P}^{1}$ as in Example 46 are Mori fiber spaces, as in Case (1).

If the exceptional locus of a contraction morphism is not a divisor, but say a curve $C$ on a threefold $X$, then the singularities on the image variety are such that 
the algorithm cannot be applied again 5 To avoid the problem of these "small" contractions one would like, on a threefold, to flip the curve $C$, that is to construct another threefold (with mild singularities) $X^{+}$, isomorphic to $X$ outside $C$, but such that $K_{X^{+}}$intersects positively the "transform" of $C\left(K_{X^{+}} \cdot C^{+}>0\right)$. Then one would need to show that a sequence of flips terminates.

3.3.2. The case of threefolds. Mori's result on existence of flips in 1988 [26] completed the algorithm for constructing minimal models for threefolds.

Theorem 66 (26]). Let $f: X \rightarrow Y$ be a birational morphism between normal projective threefolds, as in Case (3) of the previous theorem. Then there is a birational morphism $f^{+}: X^{+} \rightarrow Y$

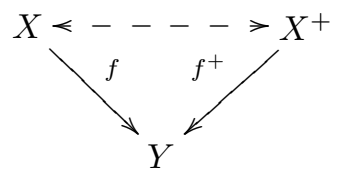

where $X^{+}$is a projective threefold with only $\mathbb{Q}$-factorial terminal singularities, $f^{+}$ contracts no divisors and $K_{X^{+}} \cdot C>0$ for any curve $C$ contracted by $f^{+}$. The birational map between $X$ and $X^{+}$is an isomorphism in codimension 1.

Definition 67. A divisor $D$ is $f$-ample if $D \cdot C>0$, for any curve $C$ contracted by $f$. The birational map between $X$ and $X^{+}$in the above theorem is called a flip.

This gives the Minimal Model Algorithm for threefolds:

Let $X$ be a threefold with $\mathbb{Q}$-factorial terminal singularities:

If $K_{X}$ is nef, STOP: $X$ is a minimal model.

If $K_{X}$ is not nef: contract any extremal ray $R$ (as in Theorem 65).

In Case (1): $X$ is a Mori fiber space: STOP.

In Case (2): replace $X$ by $Y$,

In Case (3): replace $X$ by $X^{+}$.

The process is then repeated until the canonical bundle is nef or Case (1) holds.

It turns out that neither Case (2) nor (3) can occur an infinite number of times. The termination of Case (2) follows from the fact that at every step the second Betti number decreases. The termination of Case (3) is subtle; it was shown by Shokurov; see, for example, 23.

Remark 68 . The choice of a particular extremal ray to contract is arbitrary and typically far from unique and efficient (it is easy to construct surfaces with infinitely many extremal rays). The Minimal Model Program with scaling in [3] (see Section (4) provides a "guided", efficient, contraction algorithm.

The varieties of general type are somewhat special in this regard. It turns out (in the hypothesis of the cone theorem) that if a variety is of general type, then the number of extremal rays is always finite. This principle is also used in the proof of termination of a certain kind of flips in [3]; see also Section 4.

\footnotetext{
${ }^{5}$ It is easy to see that one would get the contradiction $0=K_{X} \cdot[R]<0$.
} 
3.3.3. Log minimal pairs. There exists also a log version of the Minimal Model Program:

Let $(X, \Delta)$ be a pair consisting of a variety $X$ and an effective $\mathbb{Q}(\mathbb{R})$-Cartier divisor in $X$. We might want to consider such a pair, for example, when we have an open variety $M$ and a compactification $X=\bar{M}$, in this case $\Delta$ is the boundary divisor (see Theorem 88). Another case is given by the elliptic fibrations, which we discuss here in the case of smooth surfaces.

Example 69. An elliptic surface is a morphism: $\pi: X \rightarrow C$, to a curve $C$ where all the fibers, except a finite number, are elliptic curves; let us also assume that $X$ is minimal, for simplicity's sake. It is then a classical result 20] that $K_{X}=\pi^{*}\left(K_{C}+\Delta\right)$, where $\Delta$ is a $\mathbb{Q}$-divisor supported on the image of the fibers of $\pi$ which are not smooth elliptic curves. The singular fibers determine the coefficients of $\Delta=\sum_{i} a_{i} \Delta_{i}$; it turns out that $0 \leq a_{i}<1$. Here the $\log$ pair $(C, \Delta)$ in dimension 1 constitues as a step towards dimension 2 .

Example 70. If $V \subset X$ is a smooth hypersurface in a smooth variety $X$, then the adjunction formula (see Proposition 25) computes the canonical divisors of $V$ in term of the log pair $(X, V): K_{V}=\left(K_{X}+V\right)_{\mid V}$.

Definition 71. A $\log$ resolution of $(X, \Delta)$ is any resolution of $X f: \widetilde{X} \rightarrow X$ such that the union of the strict transform of $\Delta$ and the exceptional locus of $f$ are supported on divisors with simple normal crossings. Let $(X, \Delta)$ be a log pair, $\Delta=\sum_{i} a_{i} \Delta_{i}$ and $f: \widetilde{X} \rightarrow X$ be a log resolution of $(X, \Delta)$; we write

$$
K_{\widetilde{X}}+\widetilde{\Delta}=f^{*}\left(K_{X}+\Delta\right) \text { and } \widetilde{\Delta}=\sum d_{j} \widetilde{\Delta}_{j} .
$$

Definition 72. We have the following:

klt: The pair is kawamata log terminal if for any (equivalently for every) log resolution $f, d_{j}<1, \forall j$.

$l c$ : The pair is log canonical if for any (equivalently for every) log resolution $f, d_{j} \leq 1, \forall j$.

$d l t$ : The pair $(X, \Delta)$ is divisorially log terminal if $0<a_{i} \leq 1$ and there is a log resolution $f$ such that $d_{j}<1$ for every exceptional divisor $D_{j}$.

plt: The pair is purely log terminal if for any $\log$ resolution $f, d_{j}<1$, for every coefficient of an exceptional divisor $D_{j}$.

The pair $(C, \Delta)$ in the base of the elliptic fibration described in Example 69 is $k t l$ : $C$ is taken to be smooth and $\Delta$ consists of a finite set of points, so the only condition to verify is the one on the coefficients. The pair $(X, V)$ in Example 70 is $p t l$; in fact, the notion of a plt pair was introduced in the context of induction arguments (see Definition 83). $l c$ pairs are important also in the study of moduli spaces (see Theorem 88).

Remark 73. Klt pairs are $d l t$ pairs; dlt pairs are $l c$; see also [19. There are other variations on the definitions above, which are essential for the proofs of the theorems stated in Section [4 we do not state them here and we refer the readers to [3].

Definition 74. A curve $C$ on $X$ is log-extremal if $\left(K_{X}+\Delta\right) \cdot C<0$ and $C$ is extremal in the usual sense.

We are looking for suitable models $(Y, \Gamma)$ with $\mathbb{Q}$-factorial singularities and $K_{Y}+$ $\Gamma$ nef: 
Definition 75. Let $(X, \Delta)$ be $\log$ canonical and let $\phi: X \rightarrow Y$ be a birational map such that $\phi^{-1}$ does not contract any divisors. Let $\Gamma=\phi_{*}(\Delta)$ :

$Y$ is a log terminal model for $K_{X}+\Delta$ if $\phi$ is $\left(K_{X}+\Delta\right)$-negative, $K_{Y}+\Gamma$ is $d l t$ and nef and $Y$ is $\mathbb{Q}$-factorial.

In the next section we will see the log model at work in the proof of Theorem 81

3.3.4. Relative Minimal Model Program; finite generation and flips. There is a "relative" version of the above algorithm for a morphism $f: X \rightarrow Y$. This relative version is crucial in the proofs of [3]. In these notes we only mention the following result, which connects finite generation and flips (see for example 23], Remark 30, and Theorem 64):

Theorem 76. Let $f: X \rightarrow Y$ be a small contraction.

The sheaf $R_{f}=\bigoplus_{m \in \mathbf{N}} f_{*}\left(m K_{X}\right)$ is a sheaf of finitely generated algebras if and only if the flip of $f$ exists. Then the variety $X^{+} \stackrel{\text { def }}{=} \operatorname{Proj}(R)$ and the morphism $f^{+}$ are unique.

\section{NeW RESUlts}

Several groups of people have been working on extending the (log) Minimal Model Program to arbitrary algebraic varieties, and many important results have been obtained (see the Introduction of [3]).

In the summer of $2005 \mathrm{Siu}$ [30] announced the proof of the finite generation of the canonical ring for a smooth variety of general type. Siu's proof is based on analysis and we will not discuss it here. In the fall of 2006, Birkar, Cascini, Hacon, and $\mathrm{M}^{\mathrm{c}}$ Kernan 3 posted a paper on ArXiv on the existence of minimal models in all dimensions for varieties of general type. A corollary is the finite generation of the canonical ring and the existence of flips. Their proof of the existence of minimal models for a $n$-dimensional variety of general type does not rely on the existence or termination of flips, but it is a clever roundabout induction on $n$. In this section we present an outline of the combined induction arguments in [10, 11, and 3].

The arguments of the proofs work in the relative log set up and the use of the adjunction formula in Proposition 25] is crucial.

It should be emphasized that we do not state the results in the most general form derived in the original papers. Most notably, the theorems are not stated here in the relative setting. The relative setting is, however, crucial in the induction steps; see for example the comments on the proof of Theorem 85.

Theorem 77 (8], 3, 11], 30]). Let $X$ be a smooth variety. Then the canonical ring $R\left(X, K_{X}\right)$ is finitely generated.

Theorem 78 (Corollary of Theorem [77). Let $X$ be a smooth variety of general type, then $\operatorname{Proj}\left(R\left(X, K_{X}\right)\right)=X^{\text {can }}$ is the canonical model of $X$ (see Remark 30).

Recall that the canonical model is then unique. The $\log$ statement of the finite generation is

Theorem 79. Let $X$ be a projective variety. Suppose $K_{X}+\Delta$ is klt, and $K_{X}+\Delta$ is $\mathbb{Q}$-Cartier. Then the ring

$$
R\left(X, K_{X}+\Delta\right)=\bigoplus_{m \in \mathbf{N}} H^{0}\left(\left(\left\llcorner m\left(K_{X}+\Delta\right)\right\lrcorner\right)\right.
$$


is finitely generated. Here $\left\llcorner\sum a_{i} D_{i}\right\lrcorner=\sum\left\llcorner a_{i}\right\lrcorner D_{i}$, and $\left\lfloor a_{i}\right\rfloor$ means the round down to an integer of $a_{i}$.

Theorem 80 ([3]). Let $X$ be a smooth projective variety of general type of any dimension. Then the minimal model of $X$ exists.

The $\log$ statement of the above theorem is

Theorem 81. Let $X$ be a projective variety. If $K_{X}+\Delta$ is big and $K_{X}+\Delta$ is klt, then there exists a log terminal model of $K_{X}+\Delta$.

The proofs of the above theorems are intertwined and form a circular ascending induction on the dimension of the $(\log )$ variety $(X, \Delta)$. The ascending induction on dimension 3, 11] goes roughly as follows (but there are other inductions within the induction, and many delicate technical points, not mentioned here):

$$
\text { Th. } 79 \text { in } \operatorname{dim} n \Leftarrow \text { Th. } 81 \text { in } \operatorname{dim} n \Leftarrow \text { Th. 79] in } \operatorname{dim} n-1 \text {. }
$$

The proofs of 81 and 79 are different from the one outlined in the previous sections for the Minimal Model Algorithm; in particular the termination and existence of flips is not a building block of the proof.

In Sections 4.1 .1 and 4.1 .2 below we discuss some ideas involved in the proof of the arrows in (4.1).

\subsection{Some ideas in the proofs.}

4.1.1. Existence of log minimal models for log varieties of general type implies finite generation for the log canonical ring.

$$
\text { Theorem[79] in } \operatorname{dim} n \Leftarrow \text { Theorem } 81 \text { in } \operatorname{dim} n
$$

(Theorem 77 in $\operatorname{dim} n$ follows from Theorem 79).

Case 1. $\kappa(X, \Delta) \leq 0$. Then $R\left(X, K_{X}+\Delta\right)$ is obviously finitely generated, as in Examples 27 and 28.

Case 2. $\kappa(X, \Delta)=\operatorname{dim}(X)$, that is $(X, \Delta)$ is of general type. Then by Theorem 81 $(X, \Delta)$ has a log terminal model $(\bar{X}, \bar{\Delta})$. The divisor $K_{\bar{X}}+\bar{\Delta}$ is semiample, since it is nef and big, by the base point free theorem of Kawamata and Shokurov (TheoremDefinition 64). Then $R\left(\bar{X}, K_{\bar{X}}+\bar{\Delta}\right.$ ) is finitely generated (as in Example-Theorem 29); since the log canonical ring is a birational invariant, we have $R\left(X, K_{X}+\Delta\right)=$ $R\left(\bar{X}, K_{\bar{X}}+\bar{\Delta}\right)$.

Case 3. $0 \leq \kappa(X, \Delta)<\operatorname{dim}(X)$. This case follows from the previous one, as the following holds:

Theorem 82 ([, Fujino-Mori]). Let $(X, \Delta)$ be a klt pair with $\kappa(X, \Delta) \geq 0$, then there exists a variety $Y$ and a klt $\log$ pair $\left(K_{Y}, \Delta_{1}\right)$ of general type such that $\bigoplus_{r} H^{0}\left(X, \ell r\left(K_{X}+\Delta\right)\right)=\bigoplus_{r} H^{0}\left(Y, \ell r\left(K_{Y}+\Delta_{1}\right)\right), \ell \gg 0$.

A particular case and a "model" for Theorem 82 is given by the minimal elliptic surfaces $X$ of $\kappa(X)=1$, where $K_{C}+\Delta$ is big on $C$ (see Section 3.3.3). (In general birational Iitaka's fibrations are used; see Theorem 90 .) 
4.1.2. Finite generation and existence of certain flips imply the existence of log minimal models for varieties of general type.

$$
\text { Theorem } 81 \text { in } \operatorname{dim} n \Leftarrow \text { Theorem } 79 \text { in } \operatorname{dim} n-1 \text {. }
$$

The argument uses several log versions of the Minimal Model Program, and it relies on induction on $n$. Some of the properties of the varieties of general types which have been discussed in Example-Theorem 29 and Remark 68 become crucial here.

We have seen in the previous sections that the crucial points in completing the Minimal Model Algorithm are the existence and termination of flips. The argument of [3] cleverly avoids both issues: First the problem is modified by considering a suitable log pair $K_{X}+D$ and showing that flips exist for these particular pairs, following ideas of Shokurov. These are called pre-limiting flips, or pl flips [10, [11].

The arrow of (4.2) consists of two other arrows, which are the bulk of the inductive arguments (remember that this is only a rough outline of the proofs!):

$$
\begin{aligned}
& \text { Min. mod. in } \operatorname{dim} n \text { (Th. 81) } \Leftarrow \text { Pl-flips } \exists \text { in } \operatorname{dim} n \text { ([11] }) \\
& \Leftarrow \text { Fin. gen. in } \operatorname{dim} n-1 \text { (Th. [79). }
\end{aligned}
$$

Here we assume the existence of $p l$-flips, as in [3]; note that in [10], the existence of pl-flips is proved by induction, following ideas of Kawamata, Shokurov, and Siu.

Definition 83. Let $(X, \Delta)$ a plt pair, and let $f: X \rightarrow Z$ be a projective morphism between normal projective varieties. Then $f$ is a pl-flipping contraction if $\Delta$ is a $\mathbb{Q}$-divisor and

(1) $f$ is small, of relative Picard number one,

(2) $-\left(K_{X}+\Delta\right)$ is $f$-ample 6

(3) $X$ is $\mathbb{Q}$-factorial,

(4) $S=\llcorner\Delta\lrcorner$ is irreducible and $-S$ is f-ample.

The flip of a pl-flipping contraction $f: X \rightarrow Z$ is a small projective morphism $g: Y \rightarrow Z$ of relative Picard number one, with $\Gamma=\mu_{*}(\Delta)$,

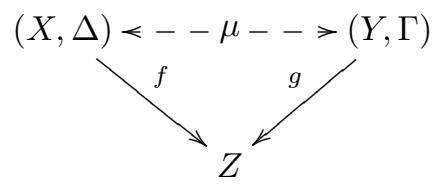

such that $K_{Y}+\Gamma$ is $g$-ample.

As in Theorem [76, the sheaf $R_{f}=\bigoplus_{m \in \mathbf{N}} f_{*}\left(m\left(K_{X}+\Delta\right)\right)$ is a sheaf of finitely generated algebras if an only if the flip of $f$ exists. The variety $Y$ and the morphism $g$ are then unique; see Remark 30, The main results of [11] imply

Theorem 84. Pl flips exist in dimension $n$.

The strategy is to consider a $k l t$ pair $(X, \Delta)$ with $\Delta \operatorname{big}, \operatorname{dim} X=n-1$, assume that the canonical ring $R(X, \Delta)$ is finitely generated and that other technical hypothesis are satisfied (in $\operatorname{dim} n-1)$. In the notation of [11] and [3, Theorem 84 (in dimension $n$ ) is Theorem $A_{n}$, while the assumptions are Theorem $F_{n-1}$.

${ }^{6}$ See Definition 67 
We now discuss some ideas in the following implication:

Theorem 81 in $\operatorname{dim} n \Leftarrow \mathrm{Pl}$-flips exist in dimension $n$.

To give a flavor of the argument used in [3, we follow the reasoning, presented in 3 . for a very particular case (recall that Theorem 80 is a particular case of Theorem 81). This will allow us to introduce some of the ideas used in the arguments: Minimal Model Program with scaling and finiteness of models combined with the induction $\operatorname{step}(s)$.

A very particular case of Theorem 80 . Assume that $X$ is a smooth variety (there is no $\log$ divisor), $K_{X}$ is big (see Section 3.2.2), and that there exists a divisor $D$ linearly equivalent to $m K_{X}$ smooth and irreducible.

Consider the pair $K_{X}+D$. It is clear that $K_{X}$ is nef if and only if $K_{X}+D$ is nef; note that $R$ is an extremal ray for $K_{X}$ if and only if it is an extremal ray for $K_{X}+D$. If $K_{X}$ is not nef, there exists an extremal ray $R$ and a contraction $\phi: X \rightarrow Z$, as in Theorem 63.

Case 1. $\phi$ is a divisorial contraction: then $K_{Z}$ is trivial and $Z$ is a minimal model.

Case 2. $\phi$ is a small contraction: $\phi$ is a small contraction for the pair $K_{X}+D$ and we are in the situation of a $p l$-flip of Definition 83 we assume that such flips exist by Theorem 84. Let $\varphi: X \rightarrow X^{\prime}$ be the flip. One still should show that any sequence of such flips terminates, but this result is not available; instead the authors of [3] run the Minimal Model Program with scaling together with induction:

The Minimal Model Program with scaling. Consider an ample divisor $H$ on $X$ and a positive number $t_{0} \in \mathbb{R}_{\geq 0}$ such that $K_{X}+D+t_{0} H$ is still ample. Then there exists a number $0 \leq t_{1} \leq t_{0}$, a ray $R$ such that $K_{X}+D+t_{1} H$ is nef and $\left(K_{X}+D+t_{1} H\right) \cdot R=0$. Note that if $t_{1}=0, K_{X}+D$ is nef and we are done. We can then assume that $0<t_{1}$, so that $\left(K_{X}+D\right) \cdot R<0$; it can be shown that we can also assume $R$ extremal. So there exists a divisorial extremal contraction or a flip, as above, and we can reiterate the algorithm. The novelty here is that the strict transforms of the divisor $\left(K_{X}+D+t_{1} H\right)$ remain nef at each step, as the algorithm is run, and this is enough to guarantee the termination of these flips. Assume in fact that the contraction of $R$ gives a flip: we restrict to $D$ and use induction. Then $\left(K_{X}+D\right)_{\mid D}=K_{D}$ (by adjunction, see Proposition 25); let $G=H_{\left.\right|_{D}}$. We have the pair $K_{D}+t_{1} G$ on $D$, with $\operatorname{dim}(D)=n-1$. Termination is proved by showing the finiteness of the possible nef pairs $\left\{K_{D}+\tau G\right\}$ that one would get by running this algorithm; the hypothesis of $K_{X}$ of general type is used (see Remark 68). A delicate point is that these minimal models are not necessarily log terminal, so a result for broader singularities is needed.

In the notation of [3], the "finiteness of models" is Theorem $E_{n-1}$; Theorem 81 is Theorem $C_{n}$. Theorem $E_{n-1}$ is also proved by induction, assuming Theorem $C_{n-1}$.

Quite a few subtleties and difficulties occur in the general case and the relative case of the Minimal Model Program needs to be considered.

\section{REMARKS}

5.1. Other results and applications. The results stated in the previous section have generated a cascade of other new results; here we mention only a few.

Theorem 85. Klt flips exist. 
The existence of flips in fact follows from a relative version of the finite generation; see also Theorem 76 .

Another result is Theorem 87 below, which describes how two different minimal models are related. In fact the minimal models in $\operatorname{dim} \geq 3$ are not necessarily unique (see Example 86). Note that if $X$ is a minimal model and $K_{X}$ is ample (then $X$ is of general type), then the minimal model is unique (we know also that $\left.X=\operatorname{Proj}\left(R\left(X, K_{X}\right)\right)\right)$.

Example 86. Let $\bar{X}=\{x y+z w=0\} \subset \mathbb{C}^{4} ; \bar{X}$ is singular at the origin. Now consider the threefolds $X \subset \mathbb{C}^{4} \times \mathbb{P}^{1}$ and $X^{\prime} \subset \mathbb{C}^{4} \times \mathbb{P}^{1}$ defined by the equations $\left\{y_{0} w+y_{1} y=0, y_{0} x+y_{1} z=0\right\}$ and $\left\{y_{0} w+y_{1} x=0, y_{0} y+y_{1} z=0\right\}$, respectively, with $\left[y_{0}, y_{1}\right] \in \mathbb{P}^{1} . X$ and $X^{\prime}$ are smooth and the natural projection morphisms $\phi: X \longrightarrow \mathbb{C}^{4}$ and $\phi^{\prime}: X^{\prime} \longrightarrow \mathbb{C}^{4}$ are isomorphisms outside the origin of $\mathbb{C}^{4}$. The composition $\varphi=\phi \cdot \phi^{\prime-1}: X \rightarrow X^{\prime}$ is an isomorphism outside $L=\phi^{-1}(0,0,0,0)$ and $L^{\prime}=\phi^{\prime-1}(0,0,0,0)$. It can be shown that $K_{X} \cdot L=K_{X^{\prime}} \cdot L^{\prime}=0$. [Exercise.] This transformation is called a flop.

Theorem 87 ([3, 13, (2008)]). Let $X$ and $X^{\prime}$ be two minimal models and $\phi: X \rightarrow$ $X^{\prime}$ a birational map. Then $\phi$ is a composition of flops.

This result was first proved in [3] for varieties of general type. The proof in 13 ] uses the existence of $k l t$ flips (Theorem 85), the termination of a certain kind of flips proved in 3, and the algorithm of the Minimal Model Program with scaling. The statement is obtained by considering a $k l t \log$ pair $(X, D)$, where $D$ is a suitable effective divisor in $X$. Then $\phi: X \rightarrow X^{\prime}$ is shown to be a composition of klt flips for the pair. Each $k l t$ flip for the pair $\left(X_{i}, D_{i}\right)$ is also a flop for the terminal variety $X$.

The following is an application to the moduli space, and it involves $l c$ pairs.

Theorem 88 ([3]). Let $X=M_{g, n}$ be the moduli space of stable curves of genus $g$ with $n$ marked points and let $\Delta_{i}, 1 \leq i \leq k$ denote the boundary divisors. Let $\Delta=\sum a_{i} \Delta_{i}$ be a boundary. Then the pair $(X, \Delta)$ is lc, and if $K_{X}+\Delta$ is big, then there is a log canonical model.

5.2. Classification. Finally a few words on how the Minimal Model Program provides a powerful tool to understand varieties in the sense of classification. Major players are also the Mori fibrations, the Iitaka fibrations, and canonical models. We have already seen this in the special case of surfaces.

The Minimal Model Program with scaling provides the following.

Theorem $89([3])$. Let $(X, \Delta)$ be a klt $\mathbb{Q}$-factorial pair. If $K+\Delta$ is not pseudoeffective, then there exists a birational map $X \rightarrow \bar{X}$ and a Mori fiber space $\pi$ : $\bar{X} \rightarrow B$.

Here we do not discuss Mori fiber spaces. We have seen that the surfaces in this class (see Theorem 34) are birationally ruled, in particular they are covered by rational curves. It is not known if the same is true in all dimensions. We refer to [9, 24], 18] for an introduction to Mori fiber spaces and several results.

For the variety of positive Kodaira dimension the following holds.

Theorem 90 (The Iitaka fibration, [34]). If $\kappa(X)>0$, there exists an integer $m \gg 0$ and a fibre space $I_{X}: \tilde{X} \rightarrow X^{\text {can }}$ which is birationally equivalent to the rational maps $\phi_{\left|m K_{X}\right|}: X \rightarrow W_{m}$, with $\operatorname{dim} W_{m}=\operatorname{dim} X^{\text {can }}=\kappa(X)$ and the general fiber $F$ of $I_{X}$ has $\kappa(F)=0$. 
Note that Theorem 77 implies $W_{m}=\operatorname{Proj}\left(R\left(X, K_{X}\right)\right)=X^{\text {can }}, \forall m \gg 0$; note also that the all the relevant $W_{m}$ are isomorphic but are given in different projective embeddings. The theorem above states that a variety $X$ of Kodaira dimension $0<\kappa(X)<\operatorname{dim}(X)$ is birationally fibered by varieties $F$ of smaller dimension and $\kappa(F)=0$.

The statement of the theorem is trivial for $\kappa(X)=0$, but formally true. If $\kappa(X)=\operatorname{dim}(X)$, and $X$ is of general type, then $X^{\text {can }}$ is the canonical model of $X$.

It is then important to know under which conditions the canonical divisor is semiample and to find the lowest $m$ such that $m K_{X}$ is base point free; this an active area of current study. Recall that if $X$ is minimal and of general type, then $K_{X}$ is semiample by Theorem-Definition 64 .

Conjecture 91 (Abundance). Let $X$ be a minimal model of a variety with $\kappa(X) \geq$ 0 . Then the canonical divisor $K_{X}$ is semiample, and it defines a morphism $\phi_{\left|m K_{X}\right|}$ : $X \rightarrow B$, for $m \gg 0$, where $B=X^{\text {can }}$ and $\kappa(F)=0$, where $F$ is the general fiber of $\phi$.

Conjecture 92. Let $X$ be a smooth projective variety. Then

a: If $\kappa(X)<0$, then there exists a variety $Y$ birational to $X$ and a morphism $f: Y \rightarrow B$ that gives $W$ the structure of Mori fiber space.

b: If $\kappa(X) \geq 0$, then $X$ is birationally equivalent to a minimal model $M$.

Let us combine the above theorems in the case of $\operatorname{dim} X=3$ (the abundance conjecture holds):

Example 93. Let $X$ be a smooth projective variety, with $\operatorname{dim} X=3$.

a: If $\kappa(X)<0$, then there exists a variety $Y$ birational to $X$ and a morphism $f: Y \rightarrow B$ which gives $W$ the structure of Mori fiber space.

b: If $\kappa(X)=0$, there is an explicit birational classification of minimal models.

c: If $\kappa(X)=1,2$, then there exists a morphism $f: M \rightarrow X^{\text {can }}$, where $M$ is the minimal model of $X$; furthermore the fibers $F$ have $\operatorname{dim} 2,1$, respectively, with $\kappa(F)=0$ (as in Theorem 90).

d: If $\kappa(X)=\operatorname{dim}(X)$, then $X$ is of general type, and the minimal model $M$ is the terminal resolution $M \rightarrow X^{\text {can }}$ of the canonical model.

\section{Summary/Timeline}

$\sim$ 1850s: $\operatorname{dim}(X)=1$. [Riemann et al.] Each smooth curve $X$ has a unique smooth minimal model, the same curve $X$.

$\sim$ 1900-1950: $\operatorname{dim}(X)=2$. [Castelnuovo-Enriques] If $\kappa(X) \geq 0$, then $X$ has a unique minimal model. If $\kappa(X)<0$, then $X$ is birational to a Mori fiber space.

1960: [Severi, Kodaira, Mumford, Bombieri et al.] A classification exists.

$\sim$ 1980s: $\operatorname{dim}(X)=3$. [Kawamata, Kollár, Mori, Reid, Shokurov et al.] If $\kappa(X) \geq 0$, then $X$ has a minimal model, unique in codimension 2.

If $\kappa(X)<0$, then $X$ is birational to a Mori fiber space.

For $\operatorname{dim} X \leq 3$, a finer structure exists.

1990s-2005: Many important results (see Introduction of [3]).

2005-2008: [Birkar, Cascini, Hacon, $M^{c}$ Kernan, et al.] dim $X$ any, $X$ of general type, then minimal model exists. 
2005-2008: [Siu; Birkar, Cascini, Hacon, $\mathrm{M}^{\mathrm{c}}$ Kernan, et al.] Let $X$ be a smooth variety, then the canonical ring $R\left(X, K_{X}\right)$ is finitely generated.

2005-2008: [Birkar, Cascini, Hacon, $M^{c}$ Kernan, et al.] $\operatorname{dim} X$ any: Flips exist.

\section{ACKNOWLEDGMENTS}

I would like to thank F. Di Sciullo, J. M'Kernan, D. Morrison, M. Rossi, J. Shaneson, S. Shatz, and L. Traynor for helpful comments, and in particular A. Collino for many insightful and inspiring conversations.

\section{About the AUTHor}

Antonella Grassi is currently Professor of Mathematics at the University of Pennsylvania. Her area of research is algebraic geometry with applications to theoretical physics.

\section{REFERENCES}

1. A. Beauville, Algebraic Surfaces, Cambrige University Press, 1983. MR732439 (85a:14024)

2. E. Bombieri, Canonical models of surfaces of general type, Inst. Hautes Études Sci. Publ. Math. 42 (1973), 171-219. MR0318163(47:6710)

3. C. Birkar, P. Cascini, C. Hacon, J. $\mathrm{M}^{\mathrm{c}}$ Kernan, Existence of minimal model for varieties of log general type, http://math.mit.edu/ mckernan/Papers/papers.html, July 2008.

4. F. Catanese, Canonical rings and "special" surfaces, in Algebraic Geometry, Bowdoin, 1985; Proceedings of Symposia in Pure Mathematics, 46-Part 1, A.M.S., Providence, 1987. MR 927956 (89f:14039)

5. A. Corti, P. Hacking, J, Kollár, R. Lazarsfeld, Lectures on Flips and Minimal Models, ArXiv:math.AG/0706.0494, 1-28, 2007.

6. O. Debarre, Higher-dimensional algebraic geometry, Universitext, Springer-Verlag, New York, 2001. MR.1841091 (2002g:14001)

7. F. Enriques, Le Superficie Algebriche (Italian), Nicola Zanichelli, Bologna, 1949. MR0031770 $(11: 202 \mathrm{~b})$

8. O. Fujino, S. Mori, A canonical bundle formula, J. Differential Geom. 56, no. 1, (2000), 167-188. MR1863025 (2002h:14091)

9. M. Grinenko, Birational models of del Pezzo fibrations, in Surveys in geometry and number theory: reports on contemporary Russian mathematics, 122-157, London Math. Soc. Lecture Note Ser., 338, Cambridge Univ. Press, Cambridge, 2007. MR2306142 (2008d:14022)

10. C. Hacon, J. M ${ }^{c}$ Kernan, On the existence of flips, ArXiv:math.AG/0507597, 2005.

11. C. Hacon, J. McKernan, Existence of minimal models for variety of log general type II, preprint, July 2008.

12. R. Hartshorne, Algebraic Geometry, Springer-Verlag, 1977. MR0463157(57:3116)

13. Y. Kawamata, Flops connect minimal models, ArXiv:math.AG/0704.1013 2007, 1-5.

14. Y. Kawamata, Finite generation of a canonical ring, ArXiv:math.AG/0804.315 2008, 1-45.

15. Y. Kawamata, K. Matsuda, K. Matsuki, Introduction to the minimal model problem, Proc. Sym. Alg. Geom. Sendai, 1985, Adv. Stud. Pure Math. 10, Kinokuniya, Tokyo (1985), 283360. MR946243 (89e:14015)

16. J. Kollár, Flops, Nagoya Math. J., 113 (1989), 15-36. MR.986434 (90e:14011)

17. J. Kollár et al., Flips and Abundance for Algebraic Threefolds, Asterisque 211 (1992), 21-45

18. J. Kollár, K. Smith, A. Corti, Rational and nearly rational varieties, Cambridge Studies in Advanced Mathematics, 92, Cambridge University Press, Cambridge, 2004. MR 2062787 (2005i:14063)

19. J. Kollár, S. Mori, Birational geometry of algebraic varieties. With the collaboration of C. H. Clemens and A. Corti., Cambridge Tracts in Mathematics, 134, Cambridge University Press, Cambridge, 1998. MR 1658959 (2000b:14018)

20. K. Kodaira Collected Works, vol. III, Princeton University Press, 1975. 
21. R. Lazarsfeld, Positivity in algebraic geometry I, Classical setting: line bundles and linear series, Ergebnisse der Mathematik und ihrer Grenzgebiete. 3. Folge. A Series of Modern Surveys in Mathematics, 49, Springer-Verlag, Berlin, 2004 MR2095471 (2005k:14001a)

22. R. Lazarsfeld, Positivity in algebraic geometry II, Positivity for vector bundles, and multiplier ideals, Ergebnisse der Mathematik und ihrer Grenzgebiete. 3. Folge. A Series of Modern Surveys in Mathematics, 48, Springer-Verlag, Berlin, 2004 MR2095472 (2005k:14001b)

23. K. Matsuki, Introduction to the Mori program, Universitext. Springer-Verlag, New York, 2002. xxiv+478 pp MR:1875410(2002m:14011)

24. J. M Kernan, The Sarkisov Program, http://www.mfo.de/programme/schedule/2007/40/OWR

25. S. Mori, Threefolds whose canonical bundles are not numerically effective, Ann. of Math. (2) 116 (1982), 133-176. MR662120 (84e:14032)

26. S. Mori, Flip theorem and the existence of minimal models for 3-folds, Jour. Amer. Math. Soc. 1 (1988) 117-253. MR.924704 (89a:14048)

27. D. Mumford, The canonical ring of an algebraic variety, Appendix to Zariski's paper "The theorem of Riemann-Roch for high multiples of an effective divisor on an algebraic surface", Ann. of Math. (2) 76 (1962), 612-615.

28. M. Reid, Canonical 3-folds, Journals de Geometrie Algebrique d'Angers, Juillet 1979/Algebraic Geometry, Angers, 1979, Sijthoff \& Noordhoff, Alphen aan den Rijn-Germantown, Md., 273-310, 1980. MR605348 (82i:14025)

29. M. Reid, 25 years of 3-folds-an old person's view, in Explicit birational geometry of 3-folds, A. Corti and M. Reid (eds.), CUP 2000, 313-343. A Young person's guide to canonical singularities, in Algebraic Geometry Bowdoin, Proc. Sympos. Pure Math. 46, 1, Amer. Math. Soc. Providence, 451-465, 1987. MR1798985 (2002b:14001)

30. Y.-T. Siu A general non-vanishing theorem and an analytic proof of the finite generation of the canonical ring, arXiv:math.AG/0610740

31. I. R. Shafarevich, Basic algebraic geometry, Translated from the Russian by K. A. Hirsch, Vol. 213, 1974. Springer Study Edition. Springer-Verlag, Berlin-New York, 1977. MR0366917 $(51: 3163)$

32. V.V. Shokurov, Numerical geometry of algebraic varieties, Proceedings of the International Congress of Mathematicians, Vol. 1, 2 (Berkeley, Calif., 1986), Amer. Math. Soc., Providence, RI, 672-681, 1987. MR 934269 (89g:14006)

33. V.V. Shokurov, Prelimiting flips, Trudy Mat. Inst. Steklova 240 no. Biratsion. Geom. Linein. Sist. Konechno Porozhdennye Algebry, 82-219, 2003. MR1993750 (2004k:14024)

34. K. Ueno, Classification theory of algebraic varieties and compact complex spaces, L. N. M. 439, Springer, Berlin, 1975. MR0506253 (58:22062)

35. P. M. H. Wilson, On the canonical ring of algebraic varieties, Comp. Math. Vol. 43, 3, 1981, 365-385. MR632435 (83g:14014)

36. O. Zariski, The theorem of Riemann-Roch for higher multiples of an effective divisor on an algebraic surfaces, Ann. of Math. (2) 76 (1962), 560-615. MR0141668 (25:5065)

Department of Mathematics, University of Pennsylvania, Philadelphia, Pennsylvania 19104

E-mail address: grassi@math.upenn.edu 